\title{
Quantum Modal Structure of a Trapped Atom in Evanescent Laguerre- Gaussian Beams
}

\author{
Saud A. Al-Awfi* \\ Department of Physics, Taibah University, Madina, Saudi-Arabia
}

Received: 19 Mar. 2013, Revised: 20 Jul. 2013, Accepted: 23 Jul. 2013

Published online: 1 Nov. 2013

\begin{abstract}
We investigate the quantum behavior of the neutral atoms trapped by the two-dimensional potential due to an optical vortex. Our model is based on the pure optical trapped of sodium atoms in evanescent Laguerre-Gaussian (LG) light. If the LG light is tuned to the blue of the atomic resonance line, atoms will experience repulsive potential, which push them away from the high intensity region to the low intensity region. The parameters selected in this evaluation are taken to guarantee that atoms are sufficiently cooled below the recoil limit to permit several quasi harmonics trapped quantum states.
\end{abstract}

Keywords: Optical vortex, evanescent Laguerre-Gaussian, trapped quantum states.

\section{Introduction}

The study of the optical manipulation of atoms in twisted light has led to notable advance in atom cooling and trapping [1-3]. Especially, the intense coherent light of laser has been used to cool neutral atoms down to the micro-Kelvin [4] and now even the nano-Kelvin regimes [5]. At such low temperatures, the de Broglie wavelike character of the atom becomes pronounced, making it necessary to treat the atom as wave phenomena.

Recently, there have been a reasonable number of proposals that concern with the optical confinement, where the atoms may be trapped in surface optical vortices and surface optical lattices [6-8]. There are some arguments that such confinement that may be useful to study Bose-Einstein condensate [9]. The subject matter of trapped neutral atoms in free space, in general, has also received an impetus with the realization that such a system can be used in quantum information processing [10,11].

The main aim of this work is to consider the problem of finding the Schrödinger modal for a trapped atoms in evanescent LG light, by solving Schrödinger equation. In contrast to the different waveguide types and the closed resonators, the half space system produces inadequate depth to the potential well at the same set of parameters.
Consequently the high intensity incident light or depositing a metallic thin film of a metallic film of finite thickness, deposited on the planar dielectric substrate is required to remove this obstacle $[7,8]$.

In the present work, we have used the latter producer, which is based on the surface of a metallic film, deposited on a planar dielectric substrate. The characteristic feature of this type of surface mode is the exponential decay of its electromagnetic fields with distance from the surface, in the vacuum region. Such an excitation persists as a well-defined entity even when the metallic film is only a few hundred atoms across, and the dielectric properties of the film can in practice be represented as those of a two-dimensional metallic sheet. In addition, this technique will assist to avoid the scatter diffusely process of the atom wave that causing by surface roughness at thermal velocities limit. Secondly it will minimize the effects of van deer Waals forces between the atom and the material surface that execute them to stick at low velocities limit [9].

The paper is organized as follows. In section 2 we outline the procedure leading to the evaluation of the optical forces on an atom and the trapping potential. In section 3 we present the theoretical model of this study, which is an optically trapped atom in optical vortex generated by evanescent LG. In section 4 we estimate the

\footnotetext{
* Corresponding author e-mail: alawfi99@ hotmail.com
} 
vibrational frequency of the trapped atom by using the harmonic oscillator approximation. In section 5, we derive the numerical solution of the two-dimensional Schrödinger equation involving the full effective potential leading to the precise details of the vibrational levels using sodium atom for illustration. Section 6 contains the conclusions.

\section{Optical forces}

In the presence of any excitation modes, the atom becomes subject to light-induced forces: dissipative force and dipole force that act on an atom in spatially inhomogeneous field. There are many methods to derive these optical forces, of which most likely the simplest is a semi-classical approach [11]. Let the interaction Hamiltonian term $H_{\text {int }}$ between the field and the atom be, in the dipole approximation,

$$
H_{\text {int }}=-\mu \cdot \mathbf{E}(\mathbf{R})
$$

where $\mathbf{R}(x, y, z)$ is the position operator for the atomic center of mass and $\mu$ is the atomic dipole moment operator. Ehrenfest's theorem allows one to write the expectation value of the force on the atom as

$$
\mathbf{F}=\langle\nabla(\mu \cdot \mathbf{E})\rangle
$$

where the gradient operator is taken with respect to $\mathbf{R}$. Since $\mu$ does not depend on $\mathbf{R}$, equation (2) can be rewritten as

$$
\mathbf{F}=\langle(\mu \cdot \hat{e}) \nabla \mathbf{E}\rangle
$$

where $\hat{e}$ is a unit polarization vector and $\mathbf{E}$ is the field amplitude. Assuming that the dimensions of the atomic wavepacket are small compared with the characteristic lengths over which $\mathbf{E}$ varies, we can approximate equation (3) by

$$
\mathbf{F}=\langle\mu \cdot \hat{e}\rangle \nabla \mathbf{E}
$$

and treat $\mathbf{R}$ as a classical variable. The expectation value appearing in Equation (4) involves the atomic dipole $\mu$ affected by the field and, as such, depends on $\mathbf{R}$. It can be calculated in direct way from the optical Bloch equations under a number of assumptions. If only two levels, $|g\rangle$ and $|e\rangle$, are involved in the process, the matrix element $\langle g|\mu \cdot \hat{e}| e\rangle \equiv \mu$ can be held to be real, and the expectation value

$$
\langle\mu \cdot \hat{e}\rangle=\mu\left(\rho_{g e}+\rho_{e g}\right)
$$

where $\rho_{g e}$ and $\rho_{e g}$ are atomic density-matrix elements. If $\omega_{0}=\left(E_{e}-E_{g}\right) / \hbar$ is the natural frequency of the $|g\rangle$ to $|e\rangle$ transition, then in the absence of interaction, we have $\rho_{g e} \propto$ $\exp i \omega_{0} t$ and $\rho_{e g} \propto \exp -i \omega_{0} t$. In a like manner, assuming the field $\mathbf{E}(t, \mathbf{R})$ is monochromatic, it can be written as a sum of two rapidly varying complex exponentials,

$\mathbf{E}(t, \mathbf{R})=\frac{1}{2} \mathbf{E}(\mathbf{R})\{\exp [-i \omega t+i \theta(\mathbf{R})]+\exp i \omega t-i \theta(\mathbf{R})\}$ where the phase factor $\theta$ may be a function of $\mathbf{R}$ and $\mathbf{E}(\mathbf{R})$ is a real amplitude. Making the rotating wave approximation in equation (4), yields

$$
\mathbf{F}(\mathbf{R})=\frac{1}{2} \mu \rho_{g e}[\nabla \mathbf{E}(\mathbf{R})+i \mathbf{E}(\mathbf{R}) \nabla \theta(\mathbf{R})] \exp [-i \omega t+i \theta(\mathbf{R})]+c . c
$$

The optical Bloch equations for a two-level atom at the point $\mathbf{R}$, with the interaction of equation (1) and the field of equation (6), are easily written down. For an atom at rest, and for time longer than $1 / \Gamma$ (where $\Gamma$ is the decay emission rate of the upper level), one can substitute into equation (7) the steady state solution

$\rho_{g e}=\frac{\Omega(\mathbf{R})}{\Gamma^{2}(\mathbf{R})+4 \Delta^{2}(\mathbf{R})+2 \Omega^{2}(\mathbf{R})}(2 \Delta(\mathbf{R})+i \Gamma(\mathbf{R})) \exp i[\omega t-\theta(\mathbf{R})]$

where $\Delta=\omega-\omega_{0}$ is the laser detuning and $\Omega(\mathbf{R})=\mu E(\mathbf{R}) / \hbar$ is the Rabi frequency associated with the incoming field. Substituting this into equation (7) yields the of optical forces

$\mathbf{F}(\mathbf{R})=\hbar \frac{\left[\Gamma(\mathbf{R}) \Omega^{2}(\mathbf{R}) \nabla \theta(\mathbf{R})-\Delta(\mathbf{R}) \nabla \Omega^{2}(\mathbf{R})\right]}{\Gamma^{2}(\mathbf{R})+4 \Delta^{2}(\mathbf{R})+2 \Omega^{2}(\mathbf{R})}=\mathbf{F}_{\text {diss }}(\mathbf{R})+\mathbf{F}_{\text {dip }}(\mathbf{R})$

The first term is the dissipative force $\mathbf{F}_{\text {diss }}(\mathbf{R})$, which acts in the direction of the gradient field phase factor $\theta$ and responsible for the parallel atomic motion. The second term in this expression is the dipole force $\mathbf{F}_{\text {dip }}(\mathbf{R})$, which acts in the direction of the gradient of the field intensity, that is, either toward low field intensity, if the detuning is positive (blue-detuned $\Delta>0$ ) or toward high intensity if it is negative (red-detuned $\Delta<0$ ). This means that the dipole force $\mathbf{F}_{\text {dip }}(\mathbf{R})$ is responsible for the normal atomic motion. The dipole force $\mathbf{F}_{d i p}(R)$ can, in general, be derived form an effective potential :

$$
\mathbf{U}(\mathbf{R})=\frac{\hbar \Delta(\mathbf{R})}{2} \ln \left[1+\frac{2 \Omega^{2}(\mathbf{R})}{4 \Delta^{2}(\mathbf{R})+\Gamma^{2}(\mathbf{R})}\right]
$$

The ability of the effective potential $\mathbf{U}(\mathbf{R})$ to trap the atoms within the central region depends, in general, on the kind of detuning $\Delta$ and the order of excited light mode $[6,8]$. The usefulness of the effective potential $\mathbf{U}(\mathbf{R})$ for the evanescent field application (which is the mean objective of this work) is that it allows one to figure out easily what the maximum velocity $v$ of the atom can be in the direction normal to the surface, if it is to be attract or reflect.

\section{Effective potential due to an evanescent LG beam}

Consider the case of a single vortex beam with a LG beam profile, impinging from within the dielectric, at an angle of incidence $\phi$, on a metallic film as shown in Figure 1. From Maxwell's equations the electric field vector components emerge with in-plane polarization in 
these regions of the structure. These fields are then subject to boundary and phase-matching conditions and the procedure leads to the evanescent field in the vacuum region expressible in cylindrical coordinates in the form [12]

$\mathbf{E}_{k \ell p}(x, y, z>0)=\frac{2 A_{k \ell p}(x, y)}{\zeta}\left(\hat{\mathbf{x}}+i \frac{k_{\|}}{k_{z 1}} \hat{\mathbf{z}}\right) \times \exp -i\left[x \eta(\omega / c) \sin \phi+k_{z 2} d\right] \times \exp \left[k_{z 1} z\right]$

where carets denote unit vectors, $\omega$ is the frequency of the light, $\eta$ the refractive index of the dielectric, and

$\zeta=\left(1-i \frac{k_{z 2} \varepsilon_{1}}{k_{z 1} \varepsilon_{2}}\right) \cosh \left(k_{z s} d\right)+\left(\frac{k_{z s} \varepsilon_{1}}{k_{z 1} \varepsilon_{s}}-i \frac{k_{z 2} \varepsilon_{s}}{k_{z s} \varepsilon_{2}}\right) \sinh \left(k_{z s} d\right)$

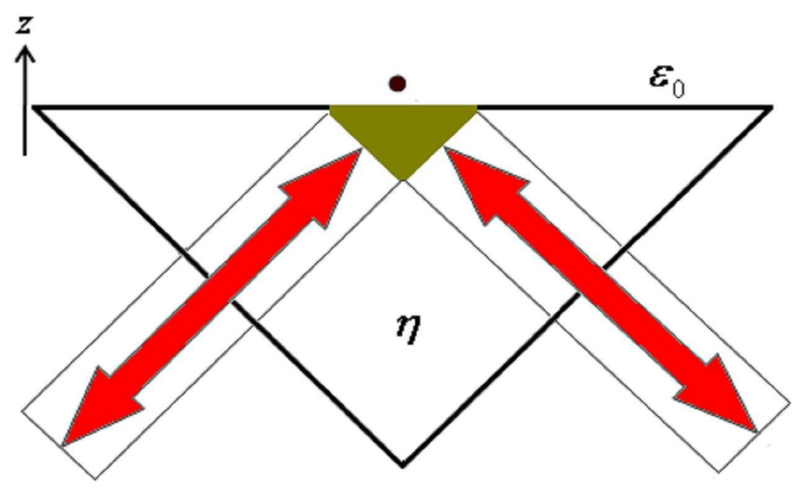

Fig. 1: Schematic total internal reflection of a two counterpropagating LG beams at planar dielectric interface with metallic film producing an evanescent mode. It is also represented an atom in a vacuum, trapped above the evanescent point.

For a $\mathrm{LG}$ beam of the order $\ell$ and degree $p$, the explicit form of the factor $A$ is given by $[7,8]$ :

$A(\mathbf{r})=C_{k \ell p}\left[\frac{\sqrt{2\left(y^{2}+(x \cos \phi)^{2}\right)}}{w_{0}}\right]^{|\ell|} \times \exp \left[\frac{-\left(y^{2}+(x \cos \phi)^{2}\right)}{w_{0}^{2}}\right]$
$\times L_{p}^{|\ell|}\left[\frac{\left(y^{2}+(x \cos \phi)^{2}\right)}{w_{0}^{2}}\right] \times \exp \left[-i \ell \arctan \left(\frac{y}{x \cos \phi}\right)\right]$

where $r=\sqrt{x^{2}+y^{2}}$ and $C_{k \ell p}$ is the normalization factor of the LG beam which is given by

$$
C_{k \ell p}=\xi_{k 00} N_{\ell p} /\left(2^{p+|\ell|} p !|\ell| !\right)^{1 / 2}
$$

In the above, $k_{\|}$is the in-plane wave-vector and $k_{Z 1}, k_{Z 2}, k_{Z S}$ are propagation wave-vectors in respective regions where the dielectric functions are $\varepsilon_{1}, \varepsilon_{2}, \varepsilon_{S}$ with regions 1 taken as vacuum and 2 the dielectric of the refractive index $\eta$, while the metallic film has a frequency-dependent dielectric function in the form
$\varepsilon_{S}=1-\omega_{p}^{2} / \omega^{2} 2$ with $\omega_{p}$ the plasma frequency. In addition the $N_{\ell p}$ is the standard coefficient $N_{\ell p}=\sqrt{p ! /(|\ell|+p !)}$ and $\xi_{k 00}$ is an electric field amplitude factor dependent on the light intensity which is given by $\xi_{k 00}=\sqrt{2 I /\left(\varepsilon_{2} \varepsilon_{0} c\right)}$. The evanescent field is thus specified in full. One should note that for the evanescent field of the type described, i.e. produced by total internal reflection at a dielectric-air interface, the penetration depth $\delta=1 / \alpha$ is essentially of the order of wavelength i.e. $\alpha=k_{\|}=2 \pi / \lambda$. This follow from [9]

$$
\delta=\frac{1}{\alpha}=\frac{2 \pi}{\lambda} \sqrt{\eta^{2} \sin ^{2} \phi-1}
$$

Consequently, if we consider an atom in the vacuum region at the position $\mathbf{R}(t)=[x(t), y(t), z(t), z>0]$. Here we think of the atom moving parallel to the film surface, rather than normal. Hence the atom is trapped to a two-dimensional space. The electronic properties of the atom are simply cast in the form of a two-level approximation with level frequency separation $\omega_{0}$ and level width $\hbar \Gamma_{0}$. The interaction of the atom with the evanescent field is taken in the dipole approximation as given in equation (1). As we mentioned above, this interaction leads to two important dynamical attributes of the motion, namely the Rabi frequency $\Omega_{k \ell p}(\mathbf{R})$ and phase $\theta_{\ell}(\mathbf{r})$. In this case, the former emerges in the following form:

$$
\begin{aligned}
& \Omega_{k \ell p}(\mathbf{R})=\frac{2 C_{k \ell p} \mu\left(1+k_{\|}^{2} / k_{z 1}^{2}\right)^{1 / 2}}{\hbar|\zeta|}\left[\frac{\sqrt{2\left(y^{2}+(x \cos \phi)^{2}\right)}}{w_{0}}\right]^{|\ell|} \\
& \times L_{p}^{|\ell|}\left[\frac{\left(y^{2}+(x \cos \phi)^{2}\right)}{w_{0}^{2}}\right] \times \exp -\left[\frac{\left(y^{2}+(x \cos \phi)^{2}\right)}{w_{0}^{2}}\right] \times \exp \left[-k_{z 1} z\right]
\end{aligned}
$$

while the phase is a function of in-plane variables:

$$
\theta_{\ell}(x, y)=\left[\ell \arctan \left(\frac{y}{x \cos \phi}\right)\right]-\left(\frac{\eta x \omega \sin \phi}{c}\right)
$$

The typical exponential decay of the evanescent effective potential field for one incidence beam given in equation (10) as shown in Figure 2 with the parameters values given in the table 1 . The potential field variations with distance normal to the surface (in units of $w_{0}$ ). This variation also depends on the values of $\ell, p$ as well as with angle of incidence $\phi$. It is seen that the length scale of the exponential decay along $z$ spans a small fraction of the wavelength $\lambda$. In consequence, the evanescent field plays an unimportant role in the trapping normal to the surface since moved atoms are subject to the much stronger attractive van der Waal's potential $[9,12]$.

The second term of phase expression $(\eta x \omega \sin \phi / c)$ is independent of the integers $\ell$ and $p$. This means that the field distribution associated with this vortex will not concentrate on the surface which gives an undesirable 
motion, as for a plane wave in free space, but one acting on the atom along the $x$-direction. This problem is formed one of the major obstacle of trapping process by single beam evanescent LG configuration. It can easily be eliminated by a simple modification to have two counter-propagating beams reflecting instead, as shown in Figure 1.

Table 1: Parameters used for the illustration of the evaluations sodium atoms in an evanescent two counter-propagating LG beams.

\begin{tabular}{||l|c|c||}
\hline Purameters & Synbol & Values Units \\
\hline \hline Wavelength & $\lambda$ & $589.0 \mathrm{~mm}$ \\
\hline Atomic mass $(\mathrm{Na})$ & $M$ & $3.8 \times 10 \mathrm{~kg}$ \\
\hline Beam waist & $w_{0}$ & $35.0 \lambda$ \\
\hline Decay rate (free space & $\Gamma \equiv \Gamma_{0}$ & $6.13 \times 10^{7} \mathrm{~s}^{-1}$ \\
\hline \hline Dipole moment & $\mu$ & $2.60 a^{\prime}$ \\
\hline Static detuning & $\Delta$ & $1.0 \times 10^{2} \Gamma$ \\
\hline \hline Field intensity & $I$ & $2 \times 10^{4} \mathrm{~W} / \mathrm{m}^{2}$ \\
\hline \hline Free space dielectric function & $\varepsilon_{1} \equiv \varepsilon_{0}$ & $\varepsilon_{1}=1.0$ \\
\hline Film dielectric function & $\varepsilon_{2}$ & 2.298 \\
\hline \hline
\end{tabular}

In this configuration, two LG beams, labeled 1 and 2, are incident at angles $\phi_{1}=-\phi_{2}=\phi$; both suffer from the internal reflection at the interface; both have field components within the film, and surface mode components in the vacuum region. We assume that the two beams are identical in the modal form, plane of incidence polarization and spatial distribution, differing only in their directions of propagation and the possibility of an associated change in the sign of the angular momentum quantum number $\ell$. The Rabi frequencies and phases may also differ in the sign of the angular momentum quantum number $\ell$. For the Rabi frequency of the evanescent field of beam 1 we write

$\Omega_{k \ell p}(\mathbf{r})=\frac{2 C_{k \ell p} \mu\left(1+k_{\|}^{2} / k_{z 1}^{2}\right)^{1 / 2}}{\hbar|\zeta|}\left[\frac{\sqrt{2\left(y^{2}+(x \cos \phi)^{2}\right)}}{w_{0}}\right]^{|\ell|}$

$\times L_{p}^{|\ell|}\left[\frac{\left(y^{2}+(x \cos \phi)^{2}\right)}{w_{0}^{2}}\right] \times \exp -\left[\frac{\left(y^{2}+(x \cos \phi)^{2}\right)^{2}}{w_{0}^{2}}\right]$

The representation of the second beam is derived from the first by rotation about the $y$-axis through an angle $(-\phi)$. Hence we can write

$$
\Omega_{2(-\phi)}^{\ell_{2}}(r)=\Omega_{1(-\phi)}^{\ell_{2}}(\mathbf{r})
$$

Consequently, the combined effect of the two beams is to give rise to effective optical potential acting on the centre
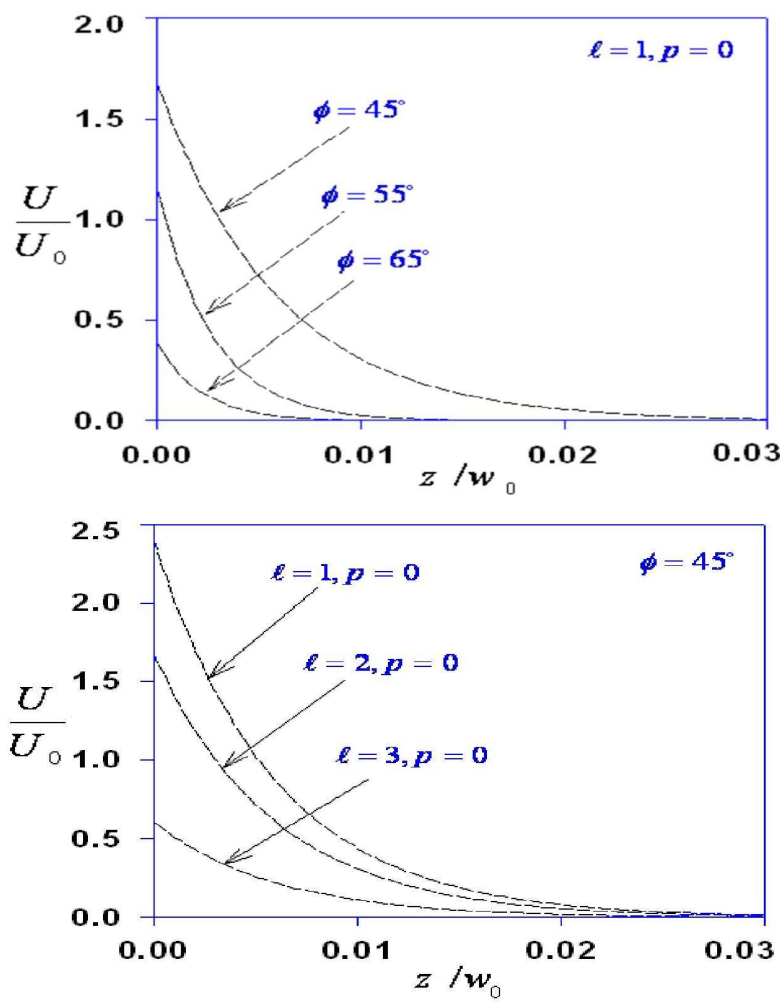

Fig. 2: The effective potential of single incidence LG beam (in units of $U_{0}=\hbar \Gamma_{0} / 2$ ) variations of the evanescent LG light with distance normal to the surface (in units of $w_{0}$ ); (a) with different angles of incidence. (b) with different orders of doughnut modes.

of mass of the atom. The new potential can be written as

$U^{1+2}(x, y)=\frac{\hbar}{2}\left[\Delta_{1 \phi} \ln \left(1+\frac{2 \Omega_{1 \phi}^{2}}{\Delta_{1 \phi}^{2}+\Gamma^{2}}\right)_{\ell_{1}}+\Delta_{1(-\phi)} \ln \left(1+\frac{2 \Omega_{1(-\phi)}^{2}}{\Delta_{1(-\phi)}^{2}+\Gamma^{2}}\right)_{\ell_{2}}\right]$

With the magnitude of the detuning as given in table 1 , we now assume $\phi_{1}=-\phi_{2}=45^{\circ}$. Figure 3 shows the variations in the xyplane of the effective potential $U^{1+2}\left(x, y, \ell_{1}=1, \ell_{2}=-1, p_{1,2}=0\right) \equiv U_{1,-1}$ generated by the evanescent field of LG light, which are created at the upper surface of the metal film by the two doughnut modes (i.e. modes with $p=0$ ) with positive detuning. The effective potential is given in units of the convenient scaling parameter $U_{0}=(1 / 2) \hbar \Gamma_{0}=3.23 \times 10^{-27} J$ and distances are measured in units of the beam waist $w_{0}$. As we seen, the effective potential is zero at the center of the laser beam and grows away from it which is an essential property of the different types of Gaussian beams. This property is referred to in literature as the fluctuating part to the dipole force and it responsible about the desirable diffusion of the attractor (reflector) atom which gives the well attractive phenomena of the optical vortex. It can be seen from Figure 3 that, the central well depth of the effective potential is enough to permit several quasi 
harmonic vibrational quantum states. The vibrational frequency of the quantum states can be estimated simply using the harmonic oscillator approximation while the precise details of these states can be obtained straightforwardly by the numerical solution of the two-dimensional Schrödinger equation involving the full effective potential.
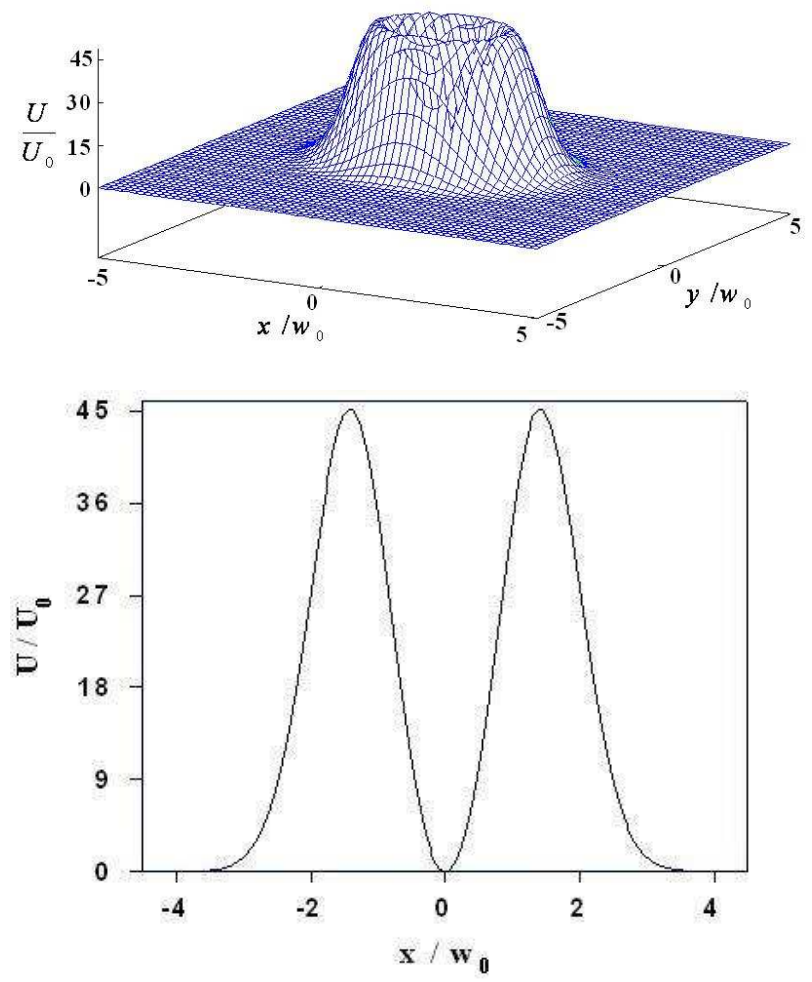

Fig. 3: $3 \mathrm{D}$ and $2 \mathrm{D}$ of the effective optical potential distribution (in unit of $U_{0}=\hbar \Gamma_{0} / 2$ ) due to evanescent two counterpropagating $\mathrm{LG}$ mode with $\ell=1, p=0$.

\section{Harmonic Oscillator Approximation}

We can estimate the vibrational frequency of the trapped atom by approximating the effective potential due to the single beam with $\ell=1, p=0$ in by the harmonic oscillator approximation around the effective potential minimum in $x y$ plane which is form a circle given by $r_{\min }=\left(x^{2}+y^{2}\right)^{1 / 2}$ as follows [13]

$$
U_{10}(\mathbf{r}) \approx U_{10}\left(\mathbf{r}_{\min }\right)+\frac{1}{2} \Lambda_{k 10}\left(\mathbf{r}-\mathbf{r}_{\min }\right)^{2}
$$

where $U_{10}\left(r_{\min }\right)$ is the effective potential depth given by

$$
U_{10}\left(\mathbf{r}_{\min }\right)=\frac{\hbar \Delta}{2} \ln \left(1+\frac{2 \Omega_{k 10}^{2}\left(\mathbf{r}_{\min }\right)}{\Delta^{2}+\Gamma^{2}}\right)
$$

and $\Lambda_{k 10}$ is an effective elastic constant given by

$$
\Lambda_{k 10}=\frac{4 \hbar|\Delta|}{\Delta^{2}+2 e^{-1} \Omega_{k 10}^{2}+\Gamma^{2}}\left(\frac{e^{-1} \Omega_{k 10}^{2}}{w_{0}^{2}}\right)
$$

The atom is considered trapped if its kinetic energy is less than $U_{\min }=3.23 \times 10^{-27} \mathrm{~J}$ and will exhibit quasi-harmonics vibrational motion about $r=r_{\min }$. The characteristic angular frequency is equal to $\sqrt{\Lambda_{k 10} / M}$ where $M$ is the atomic mass. In the two beam case, an elastic constant will be twice that for the one beam case as given in equation (23). The vibrational frequency corresponding to the parameters given in Table 1, is

$$
\omega \approx 2\left\{\frac{4 \hbar|\Delta| e^{-1} \Omega_{k 10}^{2}}{M w_{0}^{2}\left[\Delta^{2}+2 e^{-1} \Omega_{k 10}^{2}+\Gamma^{2}\right]}\right\}^{1 / 2}=1.2 \times 10^{5} \Gamma
$$

\section{The Schrödinger Equation}

From the point of view of the quantum theory, the problem of an atom immersed in an effective potential due to the evanescent field of LG light is fairly straightforward. The Schrödinger equation reads [9]

$-\frac{\hbar^{2}}{2 M}\left(\frac{\partial^{2}}{\partial r^{2}}+\frac{1}{r} \frac{\partial}{\partial r}-\frac{1}{r^{2}}-k_{\|}^{2}\right) \Psi_{n m}(r)+U_{1,-1}(r) \Psi_{n m}(r)=E \Psi_{n m}(r)$

where $E$ is the vibrational energy eigenvalue and $U_{1,-1}(r)$ is the total effective potential that given in Figure 3, while $\Psi_{n m}(r)$ is the vibrational wavefunction of the atom. One should note that, as a result of the two counter-propagate configuration the atom, is desorbed with $\vartheta_{\|} \approx 0$ which leads to that, the value of the transverse wavevector $k_{\|}$considered quantized which achieved the first condition to the quantum action. On the other hand, the second condition can be achieved by effort to make the size of effective area comparable with the thermal de Broglie wavelength $\lambda_{d B}$ of the atom which is typically a very small number (nanometers for Doppler limit cooled sodium). A micrometer-sized effective area might be the smallest one could envision making.

By considering to ease an infinite effective potential well which is a good approximation for numbers of lowest orders quantum states that give the reasonable numbers of states. The main advantage of this approximation arises first from the fact that it may provide a more extensive understanding of the quantization motion of the transverse motion of a trapping atom in a micrometers effective area of an evanescent field, in general. Second from the interesting optical properties of natural atoms that might easily be appeared in such a context. It is clear that the maximum transverse velocity $\vartheta_{\perp(\max )}$ due to the effective potential, 
which corresponds to the central potential depth can be given as [11]

$$
\vartheta_{\perp(\max )}=\left[2 U_{11}^{1+2}(r)_{\min } / M\right]^{1 / 2}
$$

Here the maximum transverse velocity $\vartheta_{\perp(\max )}$ is $0.58 \mathrm{~m} / \mathrm{s}$, therefore the momentum of the photon is analogous to the momentum of the atom or, in other words, the de Broglie atomic wavelength is large enough to observe. There are some argument should be confirmed to maintain the above analysis correctly. Originally the stability of the optical system action be able to continue via prohibited (or at least minimized) the spontaneous emission process of photons during the time $\tau$ spent by the atom in the evanescent field. This process is distinct as the product of $\Gamma \tau$ with the probability to find the atom in the excited state should be small. The latter probability scales as $\Omega^{2} /\left(\Delta^{2}+2 \Omega^{2}\right)$ for large detuning, and hence we require

$$
2 \Gamma \Omega^{2} /\left[\alpha v_{\perp}\left(\Delta^{2}+2 \Omega^{2}\right)\right] \ll 1
$$

This procedure is very important to avoid a fluctuating part of the dissipative force. On the other hand, $\tau$ has been approximate as $\tau=2 \delta / v_{\perp}=2 /\left(\alpha v_{\perp}\right)$ with the component of the atom velocity normal to the surface. The requirement condition to make the velocity dependence forces negligible is $\Gamma \gg v_{\|} / \lambda$ which could be achieved by considering a large detuning $\Delta \gg \Gamma, \Omega$. Of course, the incoming atom has component of velocity normal to surface $v_{\perp}$, such that $(1 / 2) M v_{\perp}<U_{\max }$ where maximum magnitude of total effective potential can be approximate as $U_{\max } \approx 2 \hbar \Omega^{2} / \Delta$, consequently the low spontaneous emission condition (27) yields the condition

$$
\Gamma M v_{\perp} / \alpha \hbar \Delta \ll 1
$$

This condition shows that for a given detuning it is in fact beneficial to have slowly moving atoms, even they spend more time in the evanescent field. In addition to this condition is routinely content, under the statement that $\Delta \gg \Gamma$ (where $\Delta=10^{2} \Gamma$ ), if the atom is cooled to the recoil limit (i.e. if $M v_{\perp} \approx \hbar \lambda \approx 6.2 \times 10^{-41}$ ) since $\alpha$ and $k_{\|}$are normally of the same order of magnitude. However, the Schrödinger equation (25) under these conditions can be solved numerically with high accuracy in many ways. We plot the squared modules of the first four wavefunctions of the trapped atom $\Psi_{01}, \Psi_{02}, \Psi_{11}$ and $\Psi_{12}$ in Figure 4 at the centre of the effective potential. This result is commonly accepted with the basic idea of the quantum mechanics view, in particular with problem of the infinite cylindrical well. These are precisely the similar of the well-known vibrations of an idealized circular drumhead.

For good coherent atomic beam, one would like to use the lowest order which requires the lowest transverse temperature and largest transverse de Broglie wavelength,
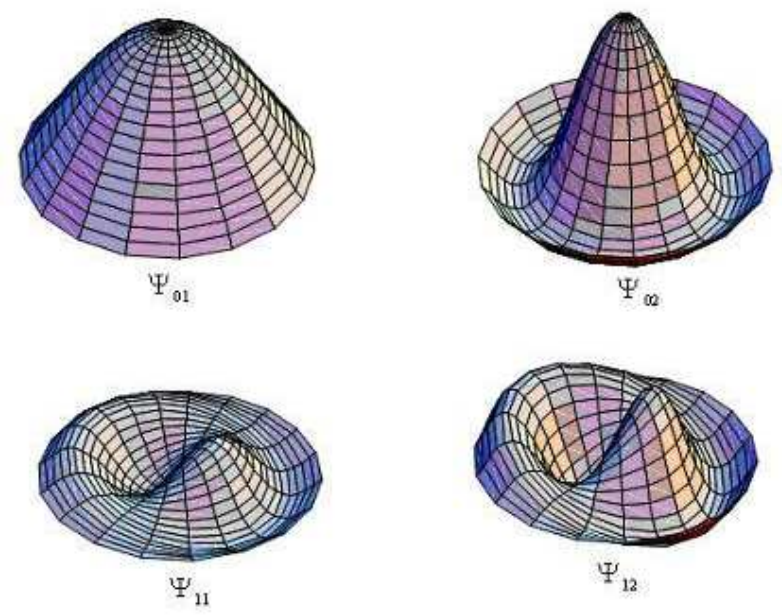

Fig. 4: First four squared modules of the atom wave functions for an atom immersed in evanescent due to two counter-propagating LG mode.

$\lambda^{\perp}=2.0 w_{0}=70.0 \lambda$. This result is commonly accepted with the basic idea of the wave optics approach, where one assumes that the wave is confined within the effective potential with a standing wave pattern in the lateral direction that falls to zero at the edge and an integral number of half-wavelengths are fitted across the cross section of the effective potential. The transverse temperature $T^{\perp}$ (in degree Kelvin) required to attain these transverse de Broglie wavelengths are given approximately by

$$
T^{\perp} \approx \frac{\hbar^{2} \pi^{2} \ell^{2}}{2 M k_{B} w_{0}^{2}}
$$

For the lowest order wave function corresponding to the parameters given in table 1, equation (29) yields transverse temperature of $T^{\perp} \approx 0.043 \mu \mathrm{K}$ which is only less by 55.8 times then the recoil temperature for sodium atom $(T=2.4 \mu K)$. However the cooling of atoms to below recoil temperature by as much as $(T / 70)$ has been achieved experimentally [5]. Useful as it is, however, the solution is still only an approximation valid in the quasi-static regime of very slowly moving atoms. Precise theoretical calculations must include velocity dependence effects and generally must be done numerically, typically as quantum trajectory calculations by using the dressed-state approach.

\section{Conclusions}

In conclusion, we have examined in detail the quantum properties of trapped atoms in the surface optical vortex generated by the evanescent Laguerre-Gaussian. The 
optical potential that acts on the atom with positive detuning to trap the atoms within the central region was evaluated. This evaluation was made using the producer based on the surface of a metallic film, deposited on a planar dielectric substrate with appropriate parameters to provide an adequately deep central well.

We have also calculated transverse atomic motion of the atom in the trapping potential due to a system mode and described this motion in terms of quantum states in two ways. First, we have estimated the vibrational frequency of these quantum states using the harmonic oscillator approximation. Second, we have evaluated them by the numerical solution of the two-dimensional Schrödinger equation.

It should be noted that, here we have neglected the effects of the gravity, but it has been considered by Harris and Savage [14]. Finally, the researcher would like to mention that the major benefit of such work arises; first from the fact that, it provides a more extensive understanding of the quantum behavior of a trapping atom immersed in the surface optical vortices and the surface optical lattices, in general. Second from the interesting optical properties of natural atoms in such a context. Additionally such work declare the evanescent wave could also be used as a quantum state selector, reflecting atoms in one quantum state secularly while scattering identical atoms in a different quantum state diffusely [9].

\section{References}

[1] L. Allen, M. J. Padgett, and M. Babiker, in Progress in Optics XXXIX, edited by E. Wolf (Elsevier Science B.V.,) (1999).

[2] L. Allen, S. M. Barnett, and M. J. Padgett, Optical Angular Momentum (PlaceTypeInstitute of PlaceNamePhysics Publishing, CityplaceBristol), (2003).

[3] Structured Light and its Applications: An Introduction to Phase-Structured Beams and Nanoscale Optical Forces, edited by D. L. Andrews (Academic, CityplaceBurlington, StateMA), (2008).

[4] Che S. and Wieman C. J. "Cooling and trapping. of atoms and ions” Opt. Soc. Am. B, 6, 122 (1989).

[5] Reichel J., Bardou F., Ben Dahan, Peik E., Rand S., Salomon C. and Cohen-Tannoudji C. Phys Rev. Lett., 75 ,4575 (1995).

[6] Al-Awfi S., Bougouffa S. and Babiker M., Opt. Commu., 283, 1022 (2009).

[7] Lembessis V., Al-Awfi S., Babiker M. and Andrews D., J. of Optics, 13, (2011).

[8] Andrews D., Babiker M., Lembessis V. and Al-Awfi S., J. Physica Status Solidi RRL (PSS), 1-3 (2010).

[9] Dowling J. and Gea-Banacloche J., Adv. Atom. Mol., 37, 1 (1996).

[10] Söding J., Grimm R. and Ovehinnikov Yu. , Opt. Commun., 119, 652 (1995).

[11] Al-Awfi S. Inter. Jour. of Mod. Phys., B 18, 10 \& 11, 1515 (2004).

[12] Kirk J., Bennett C., Babiker M. and Al-Awfi S., Phys. Low. Dim. Struct., 3/4, 127 (2002).
[13] Allen L., Babiker M., Lai W., Lembessis V, Phys. Rev., A 54, 4259 (1996).

[14] Harris D. J. and Savage C. M. Phys. Rev., A51, 3967 (1995).

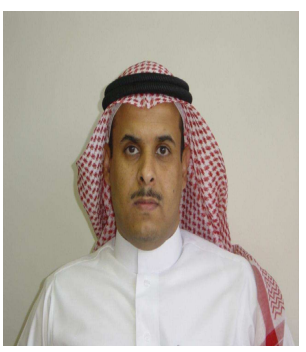

Saud A. Al-Awfi, Ms.c 1997, Ph.D. 1999, Essex University. Professor of theoretical physics at Taibah University, Faculty of Science, Department of Physics, P.O. Box 30002, Madina, Saudi Arabia. Research interests: Quantum Optics, Quantum information, Dynamics of atom with light theory, applied economics, differential geometry and applications, geometric dynamics and applications. 